\title{
Mass loss from pulsating cool stars
}

\author{
Qian Wang $^{1}$, Lee Anne Willson ${ }^{1}$ and Steven Kawaler ${ }^{1}$ \\ ${ }^{1}$ Department of Physics and Astronomy, Iowa State University, Ames, IA 50010, USA \\ email: wqinisu@iastate.edu, lwillson@iastate.edu, sdk@iastate.edu
}

\begin{abstract}
It has long been clear that most, if not all, of the mass loss experienced by stars from 0.8 to 8 solar masses occurs near the tip of the AGB and/or the RGB. Evolutionary studies have incorporated empirical mass loss laws but theoretical models suggest quite different dependence of mass loss rate on stellar parameters. We are combining evolutionary model calculations with ISUEVO with mass loss modeling using the Bowen code in a systematic study of final stages of stellar evolution. We mapped the RGB (without steady mass loss) to the "Death Zone" as a function of mixing length, mass, and metallicity. We compared these results with observation data from Origlia. We are investigating a possible mass loss mechanism through companions as a complement to mass loss through pulsation. By the end of the project we expect to provide a reliable prescription for AGB mass loss.
\end{abstract}

Keywords. Stars: mass loss, stars: AGB and post-AGB

\section{Introduction}

Stars of mass lower than 2.8 Solar mass experience an episode of mass loss that terminates the nuclear burning inside the star. Although a lot of observations support mass loss in the AGB and RGB stages, there is yet not consensus on a prescription for the mass loss rate as a function of stellar parameters. Here we report some results comparing the mass loss "death zone" derived from the Bowen models (Willson 2007a, Willson 2007b) with the location of the tip of the RGB.

From detailed models, Bowen and Willson(1991), Willson(2000), Willson(2007a), Willson(2007b) have developed a description of the mass loss process in terms of a "cliff", or deathline, and a death zone that includes most of the time that the star spends losing appreciable mass. The mass loss rate is a function of luminosity, radius, mass, and composition (and perhaps also of angular momentum and/or proximity to another star or a planet). Main sequence mass loss rate is $\leqslant 10^{-10}$ solar mass per year for single stars with $M<2.8 M_{S u n}$, leading to very small integrated mass loss before the star evolves up the red giant branch(RGB). The death line is where $\dot{\mathrm{M}}_{\text {crit }}=\mathrm{M} \dot{\mathrm{L}} / \mathrm{L}$ and the death zone extends from 0.1 to 10 times this critical mass loss rate.

We have been working on the models for RGB stars with different masses, mixing lengths and metallicities, using the stellar evolution code ISUEVO (Kawaler 2005). By comparing our models with other's work and observational data, we are trying to gain better understanding of the late stages of stellar evolution. For current results regarding AGB mass loss, see Willson(2007b).

\section{Mass loss mechanics}

After mapping the mass loss for stars on RGB tip, we find only the lowest mass, smaller mixing length (larger R) stars penetrate the death zone. For RGB mass loss, therefore, either the stars are large (mixing length parameter $\leqslant 1$ ) or RGB mass loss by the pulsation-plus-dust mechanism yields very small net mass loss on the RGB. This 
leaves two options for RGB mass loss: The mass may be lost during core He flash, dynamically or as a result of increased $\mathrm{L}$ and $\mathrm{R}$ (penetrating the death zone) or it may be lost along the RGB by some other mechanism perhaps involving some other physics not in the Bowen code. For example, the dust opacity was assumed to be grey while in reality it's not. Recently Woitke(2006) and Höfner(2007) have pointed out that there is a problem driving mass loss with non-gray silicate grains. Höfner and Anderson (Höfner 2007) suggest the solution may be in non-LTE chemistry. We will be updating the Bowen code and running a new grid of models taking this issue into account.

\section{Comparison with Tuc 47}

The Origlia et al. (2002) results show critical mass loss rates, $\dot{M} / M \sim \dot{L} / L$, over a wide range of luminosities on the RGB. This is not consistent with our understanding of pulsation-enhanced red giant winds, with or without dust. Therefore, there must be another mechanism producing this mass loss. We are investigating a mechanism involving low mass companions. To maintain $\dot{M} / M \sim \dot{L} / L$, the orbit must be expanding, i.e. mass must be leaving and not taking a lot of angular momentum with it. Because we are considering $m / M$ to be small, the giant will not be rotating and the Roche Lobe picture therefore does not apply.

\section{Conclusion}

Applying the death zone analysis from the AGB to the RGB, we find that at most modest amounts of mass can be lost from the normal mechanisms of pulsation and/or dust, and this only from the lowest mass, highest Z stars, before the core Helium flash. Thus RGB mass loss must be associated with the core He flash itself or must involve some mechanism not included in the pulsation-plus-dust Bowen models. We are investigating the effect of low mass companions as one possible mechanism for mass loss along the RGB.

\section{Acknowledgements}

We appreciate the opportunity to participate in this simulating and enjoyable conference. We also acknowledge long collaboration with G. Bowen and the use of his code for ongoing studies. NSF grant AST04-56047 to S. Ragland has supported this work; NSF grant AST0708143 is supporting further work on this project.

\section{References}

Bowen, G. H. 1988 , ApJ, 329, 299

Bowen, G. H. \& Willson, L. A. 1991, ApJ, 375, L53

Höfner, S. \& Anderson, A. 2007, Astronomy and Astrophysics, 465, L39-L42

Kawaler, S. 2005, ApJ, 621, Issue 1, pp. 432-444

Origlia, L. et al. 2002, ApJ, 571: 458-468

Struck, C. et al. 2004, MNRAS, 353, Issue 2, pp. 559-570

Sweigart, A., Greggio, L., \& Renzini, A. 1990, ApJ, 364: 527-539

Willson, L. A. 2000, ARAA, 38, pp. 573-611

Willson, L. A. 2007, Vienna proceedings, eds. Franz Kerschbaum, Corinne Charbonnel and Robert Wing, page (to appear)

Willson, L. A. 2007, this volume

Woitke, P. 2006, Astronomy and Astrophysics, 460, L9-L12 